\title{
Desenvolvimento, divulgação, adesão e eficácia de um Programa de Cessaçáo do Tabagismo oferecido em uma universidade pública
}

\author{
Fernanda Machado Lopes, Ana Carolina Wolf Baldino Peuker, Bruno Evaldt Rech, \\ Raul Gonçalves, Lisiane Bizarro
}

Universidade Federal do Rio Grande do Sul - UFRGS, Porto Alegre, RS, Brasil

\begin{abstract}
Resumo: Programas de Cessação do Tabagismo (PCT) devem integrar aspectos que aumentem as chances de sucesso do tratamento, tais como a escolha da abordagem e modalidade baseadas em evidências científicas, a adaptação da intervenção às características do público alvo, os registros dos procedimentos e dos índices de eficácia e a utilização de estratégias tanto ativas como reativas de recrutamento de fumantes. Diante disso, este estudo foi delineado com o objetivo de descrever a implantação de um PCT numa universidade pública que adotou o ambiente $100 \%$ livre da fumaça do tabaco, incluindo a avaliação dos métodos de divulgação e seu possível impacto nas taxas de adesão e sucesso do programa. Foram inscritos 128 fumantes em nove grupos oferecidos ao longo de dois anos, sendo que 97 (76\%) compareceram ao primeiro encontro, dos quais 69 (71\%) efetivamente concluíram o PCT. Os grupos que realizaram avaliação de seguimento $(n=58)$ alcançaram taxas de abstinência de $27 \%$ e $32 \%$, superiores às esperadas em tratamento unicamente psicológico e similares a índices que combinaram abordagem cognitivo-comportamental com farmacológica. As estratégias de recrutamento que combinaram estratégias ativas (contatos pessoais, convites personalizados, entrevistas individuais) e reativas (cartazes, e-mail corporativo, jornal) foram as mais eficazes para captação e adesão de fumantes ao PCT, confirmando a literatura. Conclui-se que campanhas de ambiente $100 \%$ livre da fumaça são oportunidades favoráveis para a eficácia dos PCT, que podem ser desenvolvidos e implantados por profissionais treinados de diferentes especialidades da área da saúde.
\end{abstract}

Palavras-chave: Tabagismo, Tratamento, Abandono do Hábito de Fumar.

\section{Development, dissemination, adherence, and effectiveness of a Smoking Cessation Programs offered in a public university}

\begin{abstract}
Smoking Cessation Programs (SCP) must comprise factors that increase the chances of successful treatment, such as choice of approach and modality based on scientific evidence; adaptation of the intervention to the characteristics of target patients; records of procedures and efficacy rates; and the use of both active and reactive strategies to recruit smokers. Given this, this study was designed with the aim of describing the implementation of a SCP in a public university that has adopted a $100 \%$ smoke-free campus policy, including the evaluation of publicity methods and their possible impact in adherence rates and program success. There were 128 smokers enrolled in nine program groups offered over two years: 97 (76\%) attended the first meeting and 69 (71\%) effectively completed the SCP. The groups that underwent follow-up assessment $(\mathrm{n}=58)$ achieved abstinence rates of $27 \%$ and $32 \%$, greater than expected for solely psychological treatment and similar to rates that combined cognitive-behavioral approach to pharmacology. The recruitment strategies that combined active strategies (personal contacts, personal invitations, individual interviews) and reactive strategies (posters, corporate e-mail, newspapers) were the most effective to attract and gain the adherence of smokers to the SCP, corroborating the literature. We conclude that $100 \%$ smoke-free environment campaigns are opportunities that favor the effectiveness of SCPs, which can be developed and implemented by any trained health professional.
\end{abstract}

Keywords: Smoking, Treatment, Smoking Cessation.

Autor para correspondência: Fernanda Machado Lopes, Universidade Federal do Rio Grande do Sul, Av. Paulo Gama, 110, Bairro Farroupilha, CEP 90040-060, Porto Alegre, RS, Brasil, e-mail: femlopes23@gmail.com

Recebido em 24/07/2013; $1^{a}$ Revisão em 17/02/2014; Aceito em 4/5/2014. 


\section{Introdução}

A implantação de programas de cessação do tabagismo (PCT) em instituiçôes tanto públicas como privadas constitui um desafio aos profissionais da área da saúde, uma vez que a abordagem utilizada precisa integrar os aspectos biopsicossociais envolvidos na doença. Para tanto, o envolvimento de uma equipe multiprofissional qualificada exerce papel fundamental. A dependência da nicotina é uma das mais severas, pois além de ser uma substância psicoativa que provoca alteraçốes na área relacionada à motivação, a exposição diária e em grande quantidade do fumante ao ato de fumar contribui para o aumento da dependência psicológica e comportamental. Por sua vez, a efetividade dos PCT passa a depender de questóes como a adaptação da intervençáo às necessidades e características do público alvo; das estratégias de divulgação para recrutamento dos participantes; da eficácia da abordagem e modalidade de tratamento escolhidas; e de métodos acurados de registro do padrão de consumo e da avaliação do tratamento.

O tabagismo, principal causa de morte evitável em todo o mundo, tornou-se uma pandemia de difícil controle tanto no Brasil quanto no exterior, porque não há local no mundo em que não se fume atualmente (WORLD..., 2003). Estima-se que 1,2 bilhão de pessoas sejam fumantes, o equivalente a um terço da população mundial adulta, sendo que quatro milhóes de pessoas morrem a cada ano devido a doenças relacionadas ao tabaco. No Brasil, apesar da redução na prevalência do fumo detectada pelo Instituto Nacional do Câncer (INCA) nos últimos anos (em 1989 o índice de fumantes era 32\%, em 2006 reduziu-se para 16,2\% e, em 2011, para $14,8 \%$ ), o tabaco ainda é uma substância psicoativa lícita consumida também por cerca de um terço da população (CENTRO..., 2006). Diante dessa realidade, a WHO (WORLD..., 2003) elaborou a convenção-quadro para o controle do tabagismo na qual prescreve uma série de medidas a serem adotadas pelos seus signatários com relação à produção, distribuição e venda de produtos derivados do tabaco, bem como orientações em relação ao tratamento do tabagismo. Os artigos 14.1 e 14.2 desse documento tratam de exigir medidas baseadas em evidências científicas para promover a cessação do uso do tabaco e o tratamento da dependência. Propóem a criação de programas conduzidos por profissionais especializados, que incluam procedimentos de diagnóstico e tratamento a serem realizados em instituições educativas e de saúde, bem como em espaços laborais e desportivos. As diretrizes para a implementação de tais programas sugerem a criação de uma infraestrutura que motive as tentativas de cessação e que assegure o amplo acesso a apoio para os usuários de tabaco que desejam parar de fumar (WORLD..., 2011).

No Brasil, país signatário da convenção-quadro, algumas medidas lideradas pelo Ministério da Saúde incluem a proibição de publicidade do tabaco, o aumento das alíquotas dos impostos para $85 \%$, a proibição de fumódromos, a ampliação do espaço reservado às advertências sobre os efeitos danosos do fumo nos maços e a adoção de ambientes livres da fumaça do cigarro (INSTITUTO..., 2013). Essa última é a principal recomendação da WHO, porque somente ambientes $100 \%$ livres da fumaça do tabaco oferecem proteção eficaz, já que nenhum mecanismo de ventilação ou filtragem pode reduzir a exposição à fumaça do tabaco em ambiente fechado a níveis considerados aceitáveis em termos de efeitos sobre a saúde (INSTITUTO..., 2013; PEUKER et al., 2012). Em relação ao PCT, o INCA e o Ministério da Saúde, através dos Centros Estaduais de Vigilância em Saúde (CEVS), oferecem um curso de Capacitação em Abordagem e Tratamento do Fumante para profissionais de todas as especialidades na área da saúde. O curso capacita terapeutas ocupacionais, enfermeiros, assistentes sociais, psicólogos, fisioterapeutas e nutricionistas, entre outros, para que sejam multiplicadores no desenvolvimento e implantação de PCT em seu ambiente de trabalho.

Considerando-se os prejuízos financeiros causados pelos problemas relacionados ao fumo ativo e passivo, cada vez mais empresas públicas e privadas estáo adotando a política do ambiente 100\% livre da fumaça do cigarro (CARRILLO; MAURO, 2003). Contudo, a imposição coercitiva desse tipo de política não é a estratégia mais indicada. É importante investir em campanhas de educação sobre os malefícios do fumo para conseguir a colaboração de seus funcionários para a mudança de cultura e em alternativas de tratamento. Nesse sentido, o ambiente de trabalho é um espaço propício a açóes de controle do tabagismo devido à possibilidade de se fortalecer o compromisso com a mudança de comportamento através da rede de apoio social estabelecida com colegas de trabalho (LOPES; PEUKER; BIZARRO, 2013). Além disso, a WHO (WORLD..., 2011) ressalta a importância de intervenções que tenham um cunho distinto da abordagem dos serviços de saúde e sejam realizadas também em contextos não clínicos.

Entre as intervençôes tradicionalmente mais utilizadas no tratamento do tabagismo estão o 
tratamento farmacológico, com medicação e reposição de nicotina através de adesivos transdérmicos ou gomas de mascar; e a Terapia CognitivoComportamental (TCC), referida como o tratamento não farmacológico de maior eficácia, disponibilizado pelo Ministério da Saúde à população em geral (FOCCHI; BRAUN, 2005; BRASIL, 2001). Quando comparada a outras abordagens não farmacológicas, a TCC alcançou taxas de abstinência de $15 \%$ a $25 \%$ após seis meses, bastante superiores às abordagens de aconselhamento médico (9\%), aconselhamento telefônico (10\%) e materiais de autoajuda (1\%) (PRESMAN; CARNEIRO; GIGLIOTTI, 2005). É comprovado que qualquer tratamento é mais efetivo do que nenhum, mas poucas pesquisas comparam diretamente efetividade de diferentes tratamentos não farmacológicos, tornando difícil recomendar um ou outro tipo. Contudo, o que se constata é que os fumantes que conseguem manter abstinência por mais tempo são os que fizeram múltiplas tentativas de parar e se submeteram a vários tipos de terapias simultaneamente (LANCASTER et al., 2000; PRESMAN; CARNEIRO; GIGLIOTTI, 2005).

As intervençôes grupais estão entre as formas de intervenção para dependência química mais empregadas, pois trazem consigo uma boa relaçáo custo-benefício, uma vez que oferecem a possibilidade de atender um número maior de pessoas ao mesmo tempo. Além disso, essa modalidade de tratamento permite que o indivíduo observe-se como parte integrante do grupo, possibilitando a percepção de si mesmo e do outro. Nessa abordagem, frequentemente, empregam-se conceitos de Prevenção de Recaída (para maior aprofundamento teórico ver Marlatt e Donovan (2009)), Treinamento de Habilidades Sociais (para maior aprofundamento teórico ver Zanelatto e Sakiyama (2011)) e Entrevista Motivacional (para maior aprofundamento teórico ver Miller e Rollnick (2001) e Sales e Figlie (2011)). Quanto ao modelo de estrutura, grupos abertos constituem uma boa opçáo, porque permitem a entrada de novos integrantes em qualquer fase do tratamento, beneficiando assim um maior número de pacientes de forma concomitante.

Apesar da disponibilidade de diferentes modalidades de tratamento para o tabagismo, os índices de adesão e retenção ao tratamento ainda são muito baixos, variando de $11 \%$ a $53 \%$ (AZEVEDO et al., 2008; LOPES; PEUKER; BIZARRO, 2013). O desconhecimento sobre recursos de tratamento disponíveis, a falta de informaçóes sobre o que será tratado (por exemplo, o medo da crítica e da repreensão) e o fator financeiro constituem as maiores barreiras externas à busca de auxílio especializado por fumantes que desejam parar (HUGHES; MARCY; NAUD, 2008). Outras razóes incluem a descrença quanto à eficácia da intervenção, a ideia de que não carecem de tratamento especializado para deixar de fumar e o estigma associado à busca de auxílio. Além disso, muitos fumantes receiam sofrer efeitos adversos pelo uso de medicação ou tornarem-se dependentes dos remédios (LEATHERDALE; SHIELDS, 2009). Outro aspecto que pode contribuir para os baixos índices de busca de auxílio especializado é que muitas tentativas para deixar de fumar ocorrem de forma repentina (LARABIE, 2005). Contudo, sabe-se que aqueles fumantes que recorrem a um método formal de ajuda para parar de fumar são menos suscetíveis a recaídas (HUGHES; MARCY; NAUD, 2008; LEATHERDALE; SHIELDS, 2009), por isso a importância da preparação de profissionais da área da saúde para desenvolver e implementar PCT baseados em evidências de eficácia.

Estudos epidemiológicos mostram que mais de $70 \%$ dos tabagistas desejam parar de fumar, porém menos de $10 \%$ alcançam esse objetivo por conta própria (JAIN, 2003; KHURANA et al., 2003). Assim, os profissionais da área da saúde podem ajudá-los a vencer as barreiras que os impedem de buscar auxílio informando o funcionamento do tratamento e fazendo a divulgaçáo de forma criativa e motivadora. Estudo de revisão sobre estratégias de captação de fumantes para PCT classificou as estratégias de recrutamento em três tipos: reativos, ativos e mistos. As técnicas de recrutamento reativo são aquelas que não envolvem contato pessoal direto com o público alvo, mas sim fazem a divulgação através de televisão, rádio, jornal, cartazes, folders, internet e e-mail corporativo. As estratégias ativas de recrutamento envolvem contato pessoal direto (face to face), além de contato telefônico e e-mail personalizado. Por último, as estratégias mistas são as que adotam uma combinação de técnicas ativas e reativas de recrutamento as quais, por sua vez, foram identificadas como as mais eficazes (SZKLO, 2008).

Para o tratamento, a WHO (WORLD..., 2011) ressalta o papel do aconselhamento breve, da farmacoterapia e das linhas diretas (ex. Viva Voz) nos programas de cessação do tabagismo. Ademais, estimula que se invista no uso de estratégias inovadoras, como a promoção de campanhas para o dia mundial sem tabaco e o uso de meios de comunicação alternativos, tais como mensagens de texto para celulares, publicidade em rádios comunitárias e internet. Embora ainda não haja evidências sólidas da efetividade do uso desses novos meios de intervenção, a WHO os sugere como promissores e dignos de maiores esforços em termos 
de pesquisa. Diante disso, este artigo foi delineado com o objetivo de descrever a implantação de um Programa de Cessação do Tabagismo (PCT) numa universidade pública que adotou o ambiente $100 \%$ livre da fumaça do tabaco, incluindo a avaliação dos métodos de divulgaçáo e seu possível impacto nas taxas de adesão e sucesso do programa. A complexidade desse tipo de intervenção e a escassez de relatos justificam a necessidade desse registro, promovendo a transferência desse conhecimento para o avanço de todos os profissionais de diferentes especialidades que investigam temática pertinente ao uso e abuso de álcool e outras drogas, bem como daqueles que atuam com estratégias de intervenção.

\section{Método}

Este foi um estudo quantitativo, descritivo e exploratório, de corte longitudinal. Foram realizados nove grupos do Programa de Cessação do Tabagismo (PCT) no período de setembro de 2010 a novembro de 2012. Do total de grupos oferecidos, oito foram estritamente direcionados à comunidade acadêmica, incluindo funcionários técnico-administrativos, corpos docente e discente. Devido à redução da demanda pelos grupos de cessação do tabagismo na populaçáo alvo, um grupo foi oferecido à comunidade geral.

O PCT foi desenvolvido por duas psicólogas especialistas em Psicologia Clínica, uma doutoranda capacitada pelo INCA e outra pós-doutoranda, sob supervisão de uma professora do Instituto de Psicologia da Universidade. A estrutura e o conteúdo do PCT foram concebidos a partir do material disponibilizado pelo Ministério da Saúde e Instituto Nacional do Câncer (BRASIL, 2004), complementado por técnicas da Terapia CognitivoComportamental, considerando a experiência clínica prévia das coordenadoras com pacientes dependentes de substâncias psicoativas. O PCT foi desenvolvido com o objetivo de sensibilizar os fumantes para a modificação do comportamento de fumar, oferecer psicoeducação sobre o tabagismo e treinamento de estratégias cognitivas e comportamentais para o manejo dos sintomas de abstinência e fissura.

\subsection{Participantes}

Foram inscritos 128 fumantes nos nove grupos de cessação do tabagismo oferecidos nas dependências da universidade. Desses, 97 fumantes (76\%) compareceram ao primeiro encontro, dos quais 69 (71\%) efetivamente concluíram o PCT, ou seja, participaram de pelo menos três dos quatro encontros previstos. Além disso, 58 fumantes do PCT que estavam participando de um estudo maior sobre modificação da atenção realizaram avaliação após 1,6 e 12 meses do término do grupo.

\subsection{Instrumentos}

Monoxímetro (Smokerlyser): Instrumento empregado para avaliar o nível de monóxido de carbono (CO) no ar exalado dos fumantes. Trata-se de um aparelho portátil que mede o $\mathrm{CO}$ em partículas por milhão (ppm) e em geral alcança em torno de 4 ppm no ar ambiente. Valores de 0 a 10 ppm podem ser obtidos em pessoas não fumantes, valores entre 11 e $20 \mathrm{ppm}$, em fumantes moderados, e valores entre 21 e $100 \mathrm{ppm}$, em fumantes graves (BEDFONT SCIENTIFIC, 1993).

Fagerström Test for Nicotine Dependence (FTND): Utilizado para avaliar a gravidade da dependência da nicotina (HEATHERTON et al., 1991), foi adaptado para a população brasileira em 2002 (CARMO; PUEYO, 2002). Neste estudo, escores até quatro foram considerados nível baixo de dependência; escore igual a cinco, nível moderado; e escores iguais ou superiores a seis, nível alto dependência de nicotina.

Questionários dos levantamentos: O Levantamento I foi realizado pessoalmente através de um questionário de duas perguntas: Vocêe é fumante? Você gostaria de ajuda para parar de fumar? No Levantamento II foi utilizado um questionário on-line aberto, criado em um servidor da universidade, com as seguintes perguntas: Você é fumante? Você deseja parar de fumar? Você teria interesse em participar de quatro encontros de um grupo gratuito de apoio para parar de fumar oferecido no campus onde você trabalha? Qual seu e-mail? Você gostaria de indicar alguém que teria interesse em participar?

\subsection{Procedimentos}

Os grupos foram coordenados por cinco psicólogos doutorandos e uma pós-doutoranda em formato de projeto de extensão, sendo que os dois últimos grupos contaram com apoio de um estagiário do curso de Psicologia. Os grupos foram realizados em todos os campi da universidade, em salas equipadas com recurso de data show e cadeiras universitárias. Cada encontro teve 2 horas de duração e frequência semanal, totalizando quatro encontros, sempre no turno da tarde, a pedido dos inscritos. Os participantes receberam a apostila (manual do participante) do Programa do Tratamento do Tabagismo da Rede SUS (BRASIL, 2001) e todo 
material de apoio, que incluía exercícios e técnicas comportamentais e cognitivas desenvolvidos para complementar e atender as necessidades desse público alvo. Cada encontro, a partir da segunda sessão, iniciava-se com uma breve avaliação do humor e do comportamento em relaçấo ao cigarro e com um resumo do encontro anterior para favorecer a fixação do conteúdo.

O conteúdo do protocolo aplicado no PCT (descrito em Lopes, Peuker e Bizarro (2013)) foi o mesmo nos nove grupos realizados. Contudo, o método de divulgação variou entre quatro modalidades. A Divulgação I, direcionada ao primeiro grupo, utilizou principalmente estratégia ativa de recrutamento, complementada por estratégias reativas, e foi realizada junto com a campanha para implantação do Ambiente 100\% Livre da Fumaça do Tabaco no saguáo dos prédios da reitoria da universidade. $\mathrm{Na}$ Divulgaçáo I houve um investimento maior em materiais de comunicação, como cartazes, folders e até distribuição de cataventos, símbolo adotado na campanha de ambiente $100 \%$ livre do fumo. Além disso, houve a presença da equipe da área da saúde divulgando pessoalmente a campanha e o PCT e fazendo a entrega de convites aos fumantes que desejavam ajuda para cessar (ver etapas 1 a 3).

A Divulgação II, dirigida aos grupos 2 a 7, utilizou principalmente estratégia reativa de recrutamento realizada por meio do site e da rádio da universidade, de cartazes distribuídos em todos os campi e por e-mail enviado através do setor de informática da universidade para todos os servidores e professores. Além disso, houve complemento de estratégia ativa de recrutamento, uma vez que os participantes do primeiro grupo divulgaram pessoalmente o PCT para seus colegas fumantes, e e-mails personalizados foram enviados divulgando as inscriçôes de cada grupo. $\mathrm{Na}$ modalidade de Divulgação III, direcionada ao grupo 8 , utilizou-se somente estratégia reativa de recrutamento através de comunicação geral via e-mail corporativo e cartazes produzidos por uma estagiária da área de comunicação e distribuídos nos campi centro e da saúde. Por fim, na modalidade de Divulgação IV, orientada para o único grupo (grupo 9) aberto à comunidade externa à universidade, foi empregada apenas estratégia reativa através de mídia impressa, cartazes impressos (espalhados pela universidade) e mídia social on-line (Facebook).

A seguir serão descritas as etapas realizadas neste estudo. A Tabela 1 apresenta as etapas e os métodos de divulgaçáo utilizados em cada grupo.

Etapa 1 - Campanha para implantação do Ambiente 100\% Livre da Fumaça do Tabaco (ALFT): A primeira etapa foi a consolidaçáo da parceria de três setores da universidade (Departamento de Atenção à Saúde, Instituto de Psicologia e Faculdade de Comunicação) que, com o apoio da reitoria, realizaram a campanha para implantaçáo do ALFT, a começar no prédio da reitoria e anexos. A campanha aconteceu no dia da entrada da primavera, com o slogan "Aqui bons ares circulam". Nesse contexto houve a divulgaçáo do primeiro grupo do PCT oferecido a todos os funcionários, docentes e alunos.

Etapa 2 - Levantamento I: O primeiro levantamento foi realizado pessoalmente por uma doutoranda em psicologia e uma bolsista de iniciação científica da publicidade e propaganda junto a 304 funcionários do prédio da reitoria e anexos da universidade (populaçáo de aproximadamente mil pessoas nesses prédios). Detectou-se uma prevalência de 223 funcionários não fumantes (73\%), 39

Tabela 1. Etapas e métodos de divulgação utilizados no PCT.

\begin{tabular}{|c|c|c|c|c|c|c|c|c|c|}
\hline $\begin{array}{c}\text { Etapas/Métodos de } \\
\text { divulgação }\end{array}$ & $\begin{array}{c}\text { Divulgação } \\
\text { I }\end{array}$ & & & Div & $\begin{array}{l}\text { gas } \\
\text { I }\end{array}$ & & & $\begin{array}{c}\text { Divulgação } \\
\text { III }\end{array}$ & $\begin{array}{l}\text { Divulgação } \\
\text { IV }\end{array}$ \\
\hline Grupos & 1 & 2 & 3 & 4 & 5 & 6 & 7 & 8 & 9 \\
\hline 1. Campanha ALFT & $\mathrm{X}$ & & & & & & & & \\
\hline 2. Levantamento I & $\mathrm{X}$ & & & & & & & & \\
\hline 3. Convite para o PCT & $\mathrm{X}$ & & & & & & & & \\
\hline 4. Inscrição no PCT & $\mathrm{X}$ & $\mathrm{X}$ & $\mathrm{X}$ & $\mathrm{X}$ & $\mathrm{X}$ & $\mathrm{X}$ & $\mathrm{X}$ & $\mathrm{X}$ & $\mathrm{X}$ \\
\hline 5. Entrevista individual & $\mathrm{X}$ & $\mathrm{X}$ & $\mathrm{X}$ & $\mathrm{X}$ & $\mathrm{X}$ & $\mathrm{X}$ & $\mathrm{X}$ & & \\
\hline 6. Realização do PCT & $\mathrm{X}$ & $\mathrm{X}$ & $\mathrm{X}$ & $\mathrm{X}$ & $\mathrm{X}$ & $\mathrm{X}$ & $\mathrm{X}$ & $\mathrm{X}$ & $\mathrm{X}$ \\
\hline 7. Encontros de manutenção & $\mathrm{X}$ & $\mathrm{X}$ & $\mathrm{X}$ & $\mathrm{X}$ & $\mathrm{X}$ & $\mathrm{X}$ & $\mathrm{X}$ & & \\
\hline 8. Avaliação de seguimento & $\mathrm{X}$ & $\mathrm{X}$ & $\mathrm{X}$ & $\mathrm{X}$ & $\mathrm{X}$ & $\mathrm{X}$ & $\mathrm{X}$ & & \\
\hline 9. Levantamento II & & & & & & & & $\mathrm{X}$ & \\
\hline 10. PCT para a comunidade & & & & & & & & & $\mathrm{X}$ \\
\hline
\end{tabular}

ALFT = Ambiente Livre da Fumaça do Cigarro; PCT = Programa de Cessação do Tabagismo. 
ex-fumantes (13\%) e 42 fumantes (14\%). Desses últimos, 28 assinalaram que desejavam ajuda para parar de fumar.

Etapa 3 - Convite para o PCT: Foram distribuídos pessoalmente convites personalizados para os 28 fumantes que desejavam parar de fumar, convidando-os a participarem do primeiro grupo do PCT, com informaçóes sobre o procedimento da inscrição.

Etapa 4 - Inscriçôes no PCT: As inscriçôes poderiam ser realizadas por telefone ou $e$-mail. Os interessados informavam nome, telefone e e-mail de contato e após dois dias recebiam informaçóes referentes ao grupo.

Etapa 5 - Entrevista individual: Foi realizado um contato telefônico com cada fumante inscrito agendando um horário individual para receberem explicaçóes acerca do funcionamento do PCT. Os fumantes que se voluntariaram a participar $\mathrm{da}$ pesquisa assinaram nesse momento o Termo de Consentimento Livre e Esclarecido, responderam ao FTND e sopraram o monoxímetro.

Etapa 6 - Realização do PCT: Cada grupo teve quatro encontros, com duraçáo de duas horas cada e frequência semanal. O material suplementar deste artigo apresenta um esquema completo dos tópicos, dinâmicas e técnicas utilizados em cada um dos quatro encontros do PCT.

Etapa 7 - Encontros de manutenção: Os encontros de manutenção aconteceram 30 dias após o último encontro de cada grupo e tiveram como objetivo reforçar as estratégias cognitivas e comportamentais para o manejo dos sintomas de abstinência e da fissura.

Etapa 8 - Avaliação de seguimento: Os participantes dos grupos 1 a 7 foram contatados por e-mail ou telefone e convidados a responderem novamente os instrumentos referidos após 1, 6 e 12 meses da realização de cada grupo.

Etapa 9 - Levantamento II: O questionário contendo as cinco perguntas foi enviado por e-mail através do setor de informática da universidade para todos os servidores e professores de todos os campi.

Etapa 10 - PCT para a comunidade: Depois de atendida a demanda de fumantes vinculados à universidade que desejavam ajuda para cessar o tabagismo, foi oferecida a oportunidade de participação no programa à comunidade externa.

\subsection{Análise dos dados}

Os dados foram submetidos aos procedimentos de estatística descritiva para caracterizar a amostra e também a análises de frequência. O software estatístico utilizado foi o Pacote Estatístico para Ciências Sociais (Statistical Package for the Social Sciences-SPSS) versão 18.0.

\section{Resultados}

A descrição da amostra em relação à idade, sexo e variáveis relacionadas ao comportamento de fumar foram categorizadas conforme o método de divulgação do PCT utilizado e estáo apresentadas na Tabela 2. Os dados referem-se às variáveis medidas individualmente antes da primeira sessáo do PCT.

Em relação ao perfil, a maioria dos participantes do presente estudo (excluídos os do grupo aberto à comunidade) eram funcionários (69\%) da universidade, seguidos de alunos $(26 \%)$ e professores (5\%), com média de 47 anos de idade e tempo de uso do cigarro de 31 anos. Congruente com o nível de dependência de nicotina e de monóxido de carbono no ar exalado, classificados em moderados, a média

Tabela 2. Descrição da amostra conforme método de divulgação do PCT.

\begin{tabular}{lcccc}
\hline & Divulgação I & Divulgação II & Divulgação III & Divulgação IV \\
\hline Inscritos & 14 & 83 & 15 & 16 \\
$1^{\text {a }}$ sessão & 14 & 66 & 2 & 15 \\
Participantes PCT & 11 & 46 & 1 & 11 \\
Idade (anos) & 49 & 44 & 50 & 59 \\
Sexo (F:M) & $6: 5$ & $35: 11$ & $\mathrm{M}$ & $7: 4$ \\
FTND & 5.5 & 4.7 & $\mathrm{NA}$ & $\mathrm{NA}$ \\
N $^{\circ}$ cigarros/dia & 21 & 20 & 20 & $\mathrm{NA}$ \\
Tempo que fuma & 32 & 27 & 34 & 36 \\
CO & 20 & 21 & 20 & 18 \\
\hline
\end{tabular}

Inscritos = número total de fumantes inscritos no PCT; $1^{\text {a }}$ sessão = número total de fumantes que compareceram ao primeiro encontro do grupo; Participantes = total de participantes que concluíram o PCT; F = feminino; $\mathrm{M}=$ masculino; FTND = média do nível de dependência de nicotina; Tempo que fuma = tempo médio em anos; $\mathrm{CO}=$ média do nível de monóxido de carbono por ar exalado; NA = não se aplica. 
do número de cigarros fumados por dia foi de 20 . $\mathrm{O}$ grau de escolaridade predominante foi o ensino médio completo (63\%), seguido do nível superior (31\%) - apenas $6 \%$ tinham nível de ensino inferior ao médio.

\section{1 Índices de adesão e retenção}

Na Divulgação I, realizada pessoalmente por meio de formulário escrito junto a funcionários do prédio da reitoria, 28 fumantes $(66,6 \%$ dos fumantes respondentes que ali trabalhavam) declararam-se interessados em participar do PCT. Todos esses receberam um convite personalizado, explicando o procedimento de inscrição e funcionamento. Do total, $14(50 \%)$ se inscreveram e todos compareceram no primeiro encontro, atingindo o índice máximo de adesão (100\%) no método de Divulgação I. Contudo, o índice de retenção, calculado levando-se em consideração aqueles que participaram de três ou mais dos quatro encontros do PCT, foi de 78,5\% $(\mathrm{n}=11$ pessoas).

Considerando-se o segundo método de divulgação (Divulgação II), no qual foram recrutados participantes para os grupos 2 a 7 do PCT através de e-mail, cartazes, site e rádio da universidade, os índices de adesão e retenção foram de 83\% ( $\mathrm{n}=69$ pessoas de 83 interessadas) e $66 \%$ ( $n=46$ pessoas de 69 inscritas), respectivamente. Todos os inscritos nos grupos 1 a 7 (recrutados pelos métodos de divulgação I e II) participaram de entrevista individual antes do início do programa.

O terceiro método de divulgação do PCT (Divulgaçáo III) utilizou o e-mail corporativo, por meio do qual também realizou o Levantamento II, e a distribuiçấo de cartazes pelo campus para recrutar os participantes para o grupo 8 . Em resposta ao levantamento, 60 pessoas, dentre 110 declarados fumantes, responderam que tinham interesse em parar de fumar e gostariam de participar do PCT, sendo que, desses, apenas 15 completaram o procedimento de inscriçáo. Contudo, na semana do primeiro encontro ocorreu uma greve dos servidores, de modo que apenas dois compareceram no PCT. Os demais 58 que assinalaram no levantamento vontade de participar foram contatados por e-mail e a maior parte deles justificou que não poderia participar em função da greve. Sendo assim, o índice de adesão foi de 3\% e o de retenção, de 50\% (um participante concluiu o PCT).

Os resultados dos levantamentos I e II estáo apresentados na Tabela 3.

Considerando que a demanda interna da universidade parecia ter sido atendida naquele momento, o grupo 9 foi aberto à comunidade externa. Para tanto, o método de divulgação (Divulgação IV) do PCT utilizado foi mídia impressa (jornal), divulgação de cartazes nos campus e em mídia social (Facebook). O índice de adesão foi de 94\% ( $\mathrm{n}=15$ de 16 inscritos) e o de retenção, de $73 \%$ ( $\mathrm{n}=11 \mathrm{de}$ 15 participantes).

\section{2 Índices de abstinência e redução do consumo}

Os índices de abstinência e redução, apresentados na Tabela 4, estão categorizados conforme o método de divulgação. $O$ índice de participantes que adquiriram abstinência e a mantiveram após sete dias do término do programa foi idêntico $(18,2 \%)$ nos métodos de Divulgação I (contato pessoal) e IV (jornal), mas o índice de redução do consumo do cigarro foi maior no primeiro $(45,4 \%)$, quando comparado ao segundo (36,4\%). Ainda, após um ano, o índice de abstinência dos participantes do método Divulgação I aumentou para 27,2\%.

Considerando os 47 participantes recrutados através do método de Divulgação II (e-mail, cartazes), 42 responderam à avaliação de seguimento de sete dias e 37 responderam às avaliaçôes de seis meses e um ano. $\mathrm{O}$ índice de aquisição de abstinência logo após o término do grupo foi de $19 \%$, aumentou para $29,7 \%$ após seis meses e atingiu $32,4 \%$ após um ano, excluídos os cinco que não responderam ao acompanhamento. Já o índice de redução do consumo

Tabela 3. Resultado dos levantamentos I e II.

\begin{tabular}{lcc}
\hline & $\begin{array}{c}\text { Levantamento I } \\
\text { (Pessoalmente) }\end{array}$ & $\begin{array}{c}\text { Levantamento II } \\
(\boldsymbol{E} \text {-maii) }\end{array}$ \\
\hline Total de respostas & 304 & 934 \\
Respostas completas & 304 & 799 \\
Não fumantes & $223(73 \%)$ & $530(66 \%)$ \\
Ex-fumantes & $39(43 \%)$ & $159(20 \%)$ \\
Fumantes (F) & $42(14 \%)$ & $110(14 \%)$ \\
Fumantes que querem ajuda para cessar & $28(66 \%$ dos F) & $60(54 \%$ dos F) \\
\hline
\end{tabular}


Tabela 4. Índices de abstinência e redução do consumo do cigarro após PCT.

\begin{tabular}{lcccc}
\hline & Divulgação I & Divulgação II & Divulgação III & Divulgação IV \\
\hline Abstinência após 7 dias & $2(18,2 \%)$ & $8(19 \%)$ & $1(100 \%)$ & $2(18,2 \%)$ \\
Redução após 7 dias & $5(45,4 \%)$ & $20(48 \%)$ & NA & $4(36,4 \%)$ \\
Abstinência após 6 meses & $2(18,2 \%)$ & $11(29,7 \%)$ & NA & NA \\
Redução após 6 meses & $4(36,3 \%)$ & $8(21,6 \%)$ & NA & NA \\
Abstinência após 1 ano & $3(27,2 \%)$ & $12(32,4 \%)$ & NA & NA \\
Redução após 1 ano & $4(36,3 \%)$ & $17(46 \%)$ & NA & NA \\
\hline
\end{tabular}

$\mathrm{NA}=$ não se aplica

do cigarro logo após o término do grupo foi de $48 \%$, diminuiu para $21,6 \%$ após seis meses e atingiu $46 \%$ após um ano. Tais índices foram confirmados por medição do monoxímetro, que revelou redução do CO conforme o autorrelato. Contudo, participante do grupo 8 (método Divulgação III) relatou ter parado de fumar após a terceira sessão do PCT, mas no quarto encontro seu nível de $\mathrm{CO}$ foi de 14 (fumante leve), embora seu autorrelato tenha sido de que estava se mantendo abstinente do cigarro. Náo foi realizada avaliação de seguimento os dois últimos grupos, de modo que não há parâmetros de comparação dos métodos de Divulgação III e IV com os métodos em termos de longo prazo.

\section{Discussão}

A escolha da abordagem da Terapia CognitivoComportamental (TCC) para o PCT descrito no presente estudo foi baseada na comprovação de sua eficácia, também reconhecida na orientação do Ministério da Saúde e do Instituto Nacional de Câncer (BRASIL, 2001). Essa abordagem alcançou taxas de abstinência após um ano $(27 \%$ e $32 \%$ ) superiores às esperadas nos grupos que realizaram avaliação de seguimento (grupos 1 a 7) e similares a índices que combinaram TCC com farmacologia (25\% a 32\%) (CHATKIN et al., 2004; OTERO et al., 2006). Um fator que pode ter contribuído para o sucesso no alcance da abstinência foi o desenvolvimento do PCT conforme as diretrizes da WHO para a implementação de tais programas no contexto do ambiente $100 \%$ livre da fumaça do tabaco. Ou seja, o PCT aqui descrito foi conduzido por profissionais especializados, incluiu procedimentos de diagnóstico e tratamento, foi realizado em instituição educativa e espaço laboral, com infraestrutura motivadora e com amplo acesso aos que desejassem participar (WORLD..., 2011). Além disso, os contatos telefônicos e as entrevistas individuais realizados com os participantes dos grupos $1 \mathrm{a} 7$, em função da pesquisa sobre modificação da atenção, podem ter sido, em parte, responsáveis pelas melhores proporções de abstinência neste estudo.
Esses momentos individualizados funcionaram como reforçadores da motivação de permanecer no grupo e fazer a tentativa de parar de fumar, confirmando que PCT administrados em múltiplos formatos (individual e grupal) aumentam as taxas de abstinência e devem ser encorajados (FIORE, 2000; LOPES; PEUKER; BIZARRO, 2013).

A modalidade grupal de TCC emprega as mesmas técnicas que a abordagem individual, como a combinação e o uso de intervençóes cognitivas com treinamento de habilidades comportamentais, com foco em ampliar estratégias para lidar com a síndrome de abstinência, a dependência psicológica e os condicionamentos associados ao comportamento de fumar. O tratamento em grupo proporciona algumas vantagens, como maior suporte social, maior facilitação da discussão de situaçôes de risco e meios de lidar com as mesmas e a facilitação da discussão de problemas, o que contribui para a efetividade do tratamento (MAY; WEST, 2000; PRESMAN; CARNEIRO; GIGLIOTTI, 2005). Por outro lado, o tratamento individual permite a melhor adaptação da intervenção às necessidades e características do fumante, permitindo dar atenção a possíveis comorbidades, já que a prevalência de tabagismo em pacientes portadores de transtornos psiquiátricos é mais acentuada, em comparação à população em geral (RONDINA et al., 2005). No grupo 8 do presente estudo, do qual participou apenas um integrante, foi possível direcionar o PCT às necessidades individuais, o que provavelmente contribuiu para que ele conseguisse alcançar a abstinência. Por outro lado, houve a desvantagem de não trocar experiências com o grupo de iguais, o que pode ter levado a uma recaída não revelada pelo participante, uma vez que seu nível de monóxido de carbono no ar exalado foi incongruente com o autorrelato de abstinência completa $(\mathrm{CO}=14$, nível leve). Tal situação pode ter ocorrido devido a um efeito de "desejabilidade" social, uma vez que, sozinho, o participante pode ter ficado constrangido e com medo de frustrar a terapeuta caso revelasse que não havia conseguido manter a abstinência. $\mathrm{Na}$ condução dos demais grupos, percebeu-se que a troca de experiências em relação às particularidades do 
comportamento de fumar (ex. "fico muito nervoso se sei que estou sem cigarro em casa") e às dificuldades em relação aos sintomas de abstinência (ex. "após as refeiçôes escovo os dentes imediatamente para diminuir a fissura") foi motivo de alívio de ansiedade e de aumento da motivação para fazer a tentativa de parar, conforme relato dos participantes. Da mesma forma, pareceu mais fácil que eles expusessem os momentos de fracasso nas tentativas de parada ou situaçôes de recaída, pois compartilhavam de situaçôes vividas por colegas do grupo, sendo menos influenciados pelo fator "desejabilidade" social.

$\mathrm{O}$ perfil dos fumantes no PCT foi similar em termos de idade, tempo de fumo e escolaridade ao de fumantes que procuraram um centro especializado de cessação de tabagismo em Sáo Paulo (SANTOS et al., 2008) e em ambulatório de um hospital no Ceará (SALES et al., 2006); mas com escores inferiores em termos de níveis de dependência de nicotina pelo FTND e número de cigarros fumados por dia. Estudos sugerem que sejam identificados subgrupos de fumantes para tratamento com características específicas como início precoce do tabagismo, tempo de fumo, doenças que sofrem agravo pelo tabaco e uso de outras substâncias psicoativas (CASTRO; MATSUO; NUNES, 2010). O fato de os participantes do PCT descrito neste estudo fazerem parte da mesma instituição facilitou para que pudessem ser agrupados, à medida do possível, por tipo de vínculo (servidor/aluno) e que se criasse uma rede de apoio entre colegas de trabalho. Além disso, devido à escolaridade, foi possível reduzir a parte psicoeducativa que abordaria informações já conhecidas dos participantes e inserir técnicas comportamentais e cognitivas, como resolução de problemas, distração e cartão de enfrentamento. Tal preocupação de adaptar o material disponibilizado pelo Ministério da Saúde ao público alvo bem como de agrupá-los por tipo de vínculo podem ter contribuído para as taxas de sucesso terapêutico atingidas, que foram inferiores somente a estudos que combinaram TCC à farmacologia.

Além da eficácia do tipo de abordagem e modalidade de tratamento, bem como da diversidade das características envolvidas no hábito de fumar, o terceiro fator abordado no presente estudo que pode ter impacto direto na efetividade dos PCT são as estratégias de divulgação para recrutamento dos fumantes. Foram referidas quatro diferentes modalidades de divulgação, sendo que a Divulgação I utilizou modelo combinado, predominando o recrutamento ativo; a Divulgação II, o modelo combinado, predominando o recrutamento reativo; a Divulgação III, o modelo reativo voltado ao público interno; e a Divulgação IV, o modelo reativo voltado ao público externo. Comparando as quatro modalidades em termos de eficácia, os modelos estritamente reativos alcançaram um maior número de interessados em participar do PCT, mas os melhores modelos de divulgação em termos de adesão e redução do consumo de cigarros comprovado após o término do grupo foram os que utilizaram modelos mistos. Entre os dois modelos mistos, o que utilizou predominantemente recrutamento ativo (Divulgação I) teve maiores índices de inscrição $(50 \%$ dos interessados se inscreveram) e adesão (100\% dos inscritos compareceram à primeira sessão) quando comparado ao que utilizou predominantemente recrutamento reativo, mas os índices de reduçáo do consumo do cigarro foram similares em curto, médio e longo prazo nas duas modalidades.

As avaliaçóes de seguimento dos métodos reativos voltados ao público interno e externo náo foram realizadas, o que pode ser considerada uma importante limitação deste estudo, uma vez impossibilita a comparação do índice de redução ao longo do tempo dos participantes recrutados por esses métodos. Considera-se de extrema importância que estudos sobre processo e resultados de intervenção contem com avaliaçôes longitudinais, permitindo assim a avaliação acurada das taxas de recaída e remissão completa ao longo do tempo. Em conjunto, os resultados das estratégias de recrutamento utilizadas no presente estudo mostraram que modelos que combinam estratégias ativas e reativas são os mais eficazes, sendo que cada uma tem um melhor aproveitamento, dependendo da etapa em que é utilizada. No momento do levantamento, por exemplo, o modelo on-line parece ser o mais indicado, uma vez que tem amplo alcance, exige menos tempo e possui baixo custo. Contudo, estratégias estritamente reativas como cartazes, folders e divulgação pela internet não são suficientes para que fumantes saiam da fase da pré-contemplação (quando ainda nem pensam em parar de fumar) e dirijam-se para estágios posteriores da contemplação (quando já pensam em fazer algo para parar de fumar) ou mesmo da ação (PROCHASKA; DICLEMENTE; NORCROSS, 1992; MELO; OLIVEIRA; FERREIRA, 2006), inscrevendo-se num PCT e, menos ainda, para que compareçam aos encontros propostos.

A experiência de mais de dois anos descrita neste estudo permite considerarmos que, na etapa recrutamento de fumantes para participarem do PCT, estratégias mais ativas que envolvam contato pessoal como telefonemas diretos e convites personalizados parecem provocar um comprometimento mais acentuado do que quando esse recrutamento é feito apenas pela internet, pelo menos nesse tipo de instituição. Esses resultados corroboram achados de 
que estratégias ativas ou mistas alcançaram menor e menos diversificado número de indivíduos elegíveis para inscreverem-se no programa, mas obtiveram maior proporção de participação do que aquelas reativas (SZKLO, 2008).

Cabe ressaltar que qualquer estratégia de recrutamento, por si só, constitui-se intervenção, pois pode influenciar a motivação do fumante a fazer uma tentativa de parar (SZKLO, 2008). Contudo, para que a implantaçáo de um programa seja bem-sucedida, todas as etapas devem ser minuciosamente planejadas e avaliadas pelos profissionais das diferentes especialidades envolvidos, considerando as estratégias custoefetivas disponíveis. Entre as práticas observadas no presente estudo destaca-se o uso de novas tecnologias (surveys on-line, mídia social etc.) disponíveis para o amplo alcance do público alvo, combinadas com estratégias mais individualizadas como o contato telefônico e as entrevistas individuais explicando o funcionamento do PCT e as técnicas que seriam abordadas antes do início do grupo. Ainda, adequar o tratamento, personalizando-o às necessidades dos fumantes inscritos, pode contribuir para a maior adesão e sucesso na aquisição e manutenção da abstinência.

Por fim, entre os motivos para a cessação do fumo citados pelos participantes que buscaram tratamento, um relato recorrente foi o policiamento social e a sensação de hostilidade que percebiam ao fumar em lugares públicos. Abordagens incisivas, como a do ambiente $100 \%$ livre da fumaça do tabaco, refletem na cultura e educação de uma nova sociedade que tem aprendido a ver o cigarro como problema. Não por acaso, a idade dos participantes revela uma população capaz de comparar as dificuldades presentes a uma realidade favorecedora pregressa que já náo existe mais. Sáo pessoas com certo nível educacional que já não podem ignorar as chances de serem elas as próximas figuras estampadas nas caixas de cigarro, uma vez que o sistema de saúde os convoca a fazer parte das estatísticas. A realidade atual de efetividade em tratamentos nessa população mostra que, em termos quantitativos, os índices de sucesso ainda podem melhorar, mas em termos qualitativos os PCT exercem papel importante para a mudança de estilo de vida de cada um que consegue parar de fumar.

\section{Referências}

AZEVEDO, R. C. et al. Atenção aos tabagistas pela capacitação de profissionais da rede pública. Revista de Saúde Pública, São Paulo, v. 42, n. 2, p. 353-355, 2008. http://dx.doi.org/10.1590/S0034-89102008005000011
BEDFONT SCIENTIFIC. Operator's manual for mini and micro smokerlyzers. Upchurch: Bedfont Scientific, 1993.

BRASIL. Ministério da Saúde. Instituto Nacional de Câncer. Coordenação de Prevenção e Vigilância. Programa Nacional de Controle do Tabagismo e outros fatores de risco. Rio de Janeiro, 2001.

BRASIL. Ministério da Saúde. Instituto Nacional de Câncer. Coordenaçáo de Prevençáo e Vigilância. Abordagem de tratamento do tabagismo na Rede SUS. Rio de Janeiro, 2004.

CARMO, J. T.; PUEYO, A. A. A adaptaçáo ao português do Fagerström Test for Nicotine Dependence (FTND) para avaliar a dependência e tolerância à nicotina em fumantes brasileiros. Revista Brasileira de Medicina, São Paulo, v. 59, n. 1-2, p. 73-80, 2002.

CARRILLO, L. P.; MAURO, M. Y. Uso e abuso de álcool e outras drogas: açōes de promoção e prevençáo no trabalho. Revista de Enfermagem, Rio de Janeiro, v. 11, n. 1, p. 25-33, 2003

CASTRO, M. R.; MATSUO, T.; NUNES, S. O. Caracteristicas clínicas e qualidade de vida de fumantes em um centro de referência de abordagem e tratamento do tabagismo. Jornal Brasileiro de Pneumologia, Brasília, v. 36, n. 1, p. 67-74, 2010. http://dx.doi.org/10.1590/ S1806-37132010000100012

CENTRO BRASILEIRO DE INFORMACÃO SOBRE DROGAS PSICOTRÓPICAS - CEBRID. II levantamento domiciliar sobre o uso de drogas psicotrópicas no Brasil: estudo envolvendo as 108 maiores cidades do país. São Paulo: CEBRID; UNIFESP, 2006.

CHATKIN, J. M. et al. Abstinence rates and predictors of outcome for smoking cessation: do Brazilian smokers need special strategies? Addiction, London, v. 99, n. 6, p. 778-784, 2004. PMid:15139876. http://dx.doi. org/10.1111/j.1360-0443.2004.00755.x

FIORE, M. C. US public health service clinical practice guideline: treating tobacco use and dependence. Respiratory Care, Boston, v. 45, n. 10, p. 1200-1262, 2000.

FOCCHI, G.; BRAUN, I. Tratamento farmacológico do tabagismo. Revista de Psiquiatria Clínica, São Paulo, v. 32, n. 5, p. 259-266, 2005.

HEATHERTON, T. F. et al. The Fagerström test for nicotine dependence: a revision of the Fagerström Tolerance Questionnaire. British Journal of Addiction, London, v. 86, n. 9, p. 1119-1127, 1991. http://dx.doi. org/10.1111/j.1360-0443.1991.tb01879.x

HUGHES, J. R.; MARCY, T. W.; NAUD, S. Interest in treatment to stop smoking. Journal of Substance Abuse Treatment, Philadelphia, v. 36, n. 1, p. 18-24, 2008. PMid:18550319 PMCid:PMC2635950. http://dx.doi. org/10.1016/j.jsat.2008.04.002

INSTITUTO NACIONAL DE CÂNCER - INCA. Tabagismo. Rio de Janeiro: INCA, 2013. Disponível em: <http://www.inca.gov.br/tabagismo/frameset. asp?item=faq $>$. Acesso em: 16 jan. 2013.

JAIN, A. Treating nicotine addiction. British Medical Journal, London, v. 327, n. 7428, p. 1394-1396, 2003. PMid:14670889 PMCid:PMC292997. http://dx.doi. org/10.1136/bmj.327.7428.1394 
KHURANA, S. et al. Twenty-first century tobacco use: it is not just a risk factor anymore. Respiratory Medicine, Philadelphia, v. 97, n. 4, p. 295-301, 2003. http://dx.doi. org/10.1053/rmed.2002.1443

LANCASTER, T. et al. Effectiveness of interventions to help people stop smoking: findings from the Cochrane Library. British Medical Journal, London, v. 321, n. 7257, p. 355-358, 2000. http://dx.doi.org/10.1136/ bmj.321.7257.355

LARABIE, L. C. To what extent do smokers plan quit attempts? Tobacco Control, London, v. 14, n. 6, p. 425-428, 2005. PMid:16319368 PMCid:PMC1748114. http://dx.doi.org/10.1136/tc.2005.013615

LEATHERDALE, S. T.; SHIELDS, M. Smoking cessation: intentions, attempts and techniques. Health Report, Toronto, v. 20, p. 31-39, 2009.

LOPES, F. M.; PEUKER, A. C.; BIZARRO, L. Aplicação de um Programa de Cessação do Tabagismo com rodoviários urbanos. Psicologia: Ciência e Profissão, Brasília, v. 33, n. 2, p. 490-499, 2013. http://dx.doi. org/10.1590/S1414-98932013000200017

MARLATT, G. A.; DONOVAN, D. M. Prevenção de recaida: estratégias de manutenção no tratamento de comportamentos adictivos. Porto Alegre: Artmed, 2009.

MAY, S.; WEST, R. Do social support interventions ("buddy systems") aid smoking cessation? A review. Tobacco Control, London, v. 9, n. 4, p. 415-422, 2000. http://dx.doi.org/10.1136/tc.9.4.415

MELO, W. V.; OLIVEIRA, M. D.; FERREIRA, E. A. Estágios motivacionais, sintomas de ansiedade e de depressão no tratamento do tabagismo. Interação em Psicologia, Curitiba, v. 10, p. 91-99, 2006.

MILLER, W. R.; ROLLNICK, S. Entrevista motivacional: preparando as pessoas para a mudança de comportamentos adictivos. Porto Alegre: Artmed, 2001.

OTERO, U. B. et al. Ensaio clínico randomizado: efetividade da abordagem cognitivo-comportamental e uso de adesivos transdérmicos de reposição de nicotina, na cessação de fumar, em adultos residentes no Município do Rio de Janeiro, Brasil. Caderno de Saúde Pública, Rio de Janeiro, v. 22, n. 2, p. 439-49, 2006.

PEUKER, A. C. et al. Cessação do tabagismo no contexto da campanha UFRGS livre da fumaça do tabaco. Revista da Extensão, Porto Alegre, v. 1, p. 38-45, 2012.
PRESMAN, S.; CARNEIRO, E.; GIGLIOTTI, A. Tratamentos não-farmacológicos para o tabagismo. Revista de Psiquiatria Clínica, São Paulo, v. 32, n. 5, p. 267-275, 2005. http://dx.doi.org/10.1590/ S0101-60832005000500004

PROCHASKA, J. O.; DICLEMENTE, C. C.; NORCROSS, J. C. Search of how people change: Applications to addictive behaviors. American Psychologist, Washington, v. 47, n. 9, p. 1102-1113, 1992. http://dx.doi. org/10.1037/0003-066X.47.9.1102

RONDINA, R. C. et al. Um estudo comparativo entre características de Personalidade de Universitários fumantes, ex-fumantes e não-fumantes. Revista de Psiquiatria do Rio Grande do Sul, Porto Alegre, v. 27, n. 2, p. 140-150, 2005. http://dx.doi.org/10.1590/S0101-81082005000200004

SALES, M. P. et al. Ambulatório de apoio ao tabagista no Ceará: perfil dos pacientes e fatores associados ao sucesso terapêutico. Jornal Brasileiro de Pneumologia, Brasília, v. 32, n. 5, p. 410-417, 2006. http://dx.doi.org/10.1590/ S1806-37132006000500007

SALES, C.; FIGLIE, N. Entrevista motivacional. In: DIEHL, A.; CORDEIRO, D. C.; LARANJEIRA, R. Dependência química: prevenção, tratamento e políticas públicas. Porto Alegre: Artmed, 2011. p. 267-277.

SANTOS, S. R. et al. Perfil dos fumantes que procuram um centro de cessação do tabagismo. Jornal Brasileiro de Pneumologia, Brasília, v. 34, n. 9, p. 695-701, 2008.

SZKLO, A. S. Review of strategies to recruit smokers for smoking cessation: a population impact perspective. Caderno de Saúde Pública, Rio de Janeiro, v. 24, n. 4, p. 621-634, 2008.

WORLD HEALTH ORGANIZATION - WHO. WHO Framework Conventionon Tobacco Control. Genebra: WHO, 2003.

WORLD HEALTH ORGANIZATION - WHO. WHO Framework Convention on Tobacco Control: guidelines for implementation article 5.3, article 8, articles 9 and 10, article 11 , article 12 , article 13 , article 14 . Genebra: WHO, 2011.

ZANELATTO, N.; SAKIYAMA, H. M. T. Terapia Cognitivo-Comportamental das habilidades sociais e de enfrentamento. In: DIEHL, A.; CORDEIRO, D. C.; LARANJEIRA, R. Dependência química: prevenção, tratamento e políticas públicas. Porto Alegre: Artmed, 2011. p. 288-300. PMid:21726890.

\section{Contribuição dos Autores}

Fernanda Machado Lopes: redação do texto, organização de fontes, análise dos dados, revisão do texto. Ana Carolina Wolf Baldino Peuker, Bruno Evaldt Rech e Raul Gonçalves: redação do texto. Lisiane Bizarro: revisão do texto. Todos os autores aprovaram a versão final do texto.

\section{Fonte de financiamento}

CNPq e CAPES. 